\title{
Elastic interaction between "hard" or "soft" pointwise inclusions on biological membranes
}

\author{
Denis Bartolo ${ }^{1}$ and Jean-Baptiste Fournier ${ }^{1,2}$ \\ ${ }^{1}$ Laboratoire de Physico-Chimie Théorique, ESPCI, \\ 10 rue Vauquelin, F-75231 Paris cedex 05, France \\ ${ }^{2}$ Fédération de Recherche CNRS 2438 "Matière et Systèmes Complexes"
}

(Dated: October 29, 2018)

\begin{abstract}
We calculate the induced elastic-interaction between pointwise membrane inclusions that locally interact up to quadratic order with the membrane curvature tensor. For isotropic inclusions, we recover the usual interaction proportional to the inverse fourth power of the separation, however with a prefactor showing a non-trivial dependence on the rigidity $\Gamma$ of the quadratic potential. In the large $\Gamma$ limit, corresponding to "hard" inclusions, we recover the standard prefactor first obtained by Goulian et al. [Europhys. Lett. 22, 145 (1993)]. In the small $\Gamma$ limit, corresponding to "soft" inclusions, we recover the recent result of Marchenko and Misbah [Eur. Phys. J. E 8, 477 (2002)]. This shows that the latter result bears no fundamental discrepancy with previous works, but simply corresponds to the limit of soft inclusions. We discuss how the same inclusion can be depicted as hard or soft according to the degree of coarse-graining of the pointwise description. Finally, we argue that conical transmembrane proteins should be fundamentally considered as hard inclusions.

PACS numbers: 87.16.Dg (Membranes, bilayers, and vesicles), 87.15.Kg (Molecular interactions; membraneprotein interactions)
\end{abstract}

\section{INTRODUCTION}

In a recent paper [1], Marchenko and Misbah have revisited the calculation of the long-range, membrane-mediated interaction acting between inclusions embedded in biological or surfactant membranes. Using a method inspired from electrostatics, they calculated an "induced interaction", which they compared with the results of several works previously published on the subject [2, 3, 4, 5, 6]. They pointed out two major discrepancies: (i) their result is proportional to $1 / \kappa^{2}$ ( $\kappa$ being the bending rigidity of the membrane) while the induced interaction calculated in Refs. [2, 3, 4, 5, 6] is directly proportional to $\kappa$, (ii) the result of Refs. [2, 3, 4, 5, 6] involves the size of the inclusion (either directly or through a cutoff in the case of pointwise descriptions) whereas the size of the inclusion does not appear in the result of Ref. 1].

In this contribution, we show that there are no discrepancies between the approaches of Refs. [2, 3, 4, 5, 6] and that of Ref. 1], neither in the spirit of the model nor in the results. We demonstrate that the different expressions obtained for the induced interaction correspond to different limits of a common, more general model. We do so by solving a pointwise quadratic interaction model similar to the one used in Ref. [1], exactly, instead of perturbatively, and without the need to introduce a high-wavector cutoff. According to the rigidity $\Gamma$ of the quadratic interaction potential, we find either the standard prefactor first obtained by Goulian et al. 2, 3, 4, 5, 6], in the large $\Gamma$ limit corresponding to "hard" inclusions, or the result of Marchenko and Misbah [1], in the small $\Gamma$ limit corresponding to "soft" inclusions.

In the second part of the paper we discuss the role of the cutoff (introduced e.g. in Refs. [5, 6]) in relation with a coarse-graining procedure of the pointwise description. We determine how the effective quadratic interaction potential depends on the coarse-graining scale, and we discuss the distinction between hard and soft inclusions in the light of this analysis.

\section{INDUCED INTERACTION}

Here, we calculate the induced interaction between pointwise "defects", or inclusions. We describe the inclusions using the same formalism as in Ref. [1]. We use, however, a more general Hamiltonian to describe the elasticity of the membrane, and, in addition, we perform our calculations exactly, whereas the result of Ref. [1] was obtained at lowest-order in a perturbative expansion. We emphasize that we shall not introduce any cutoff during our calculation.

The generalized membrane elastic Hamiltonian [7] we use is

$$
F_{\mathrm{m}}=\frac{1}{2} \int d x d y\left[\gamma h^{2}+\sigma(\nabla h)^{2}+\kappa\left(\nabla^{2} h\right)^{2}+\kappa_{6}\left(\nabla \nabla^{2} h\right)^{2}+\kappa_{8}\left(\nabla^{4} h\right)^{2}\right]
$$


where $h(x, y)$ describes the normal displacement of the membrane. Being quadratic in $h(x, y)$, this Hamiltonian is accurate only for small membrane deformations. We have assumed that the membrane has no physical boundary except at infinity, which allowed us to discard all the terms that could be written as the divergence of a vector field (e.g., the Gaussian curvature energy [ $]$ ). The parameters $\gamma$ and $\sigma$ describe a confining potential and a membrane tension, respectively. In most cases they are negligibly small, and they play no role except at very large scales. The parameter $\kappa$ is the usual bending constant [7] and $\kappa_{6}, \kappa_{8}$ are higher-order bending elasticity constants [8]. We may rewrite the membrane Hamiltonian in the reciprocal space as

$$
F_{\mathrm{m}}=\int \frac{d^{2} q}{(2 \pi)^{2}} \frac{\kappa}{2}\left[\ell_{\gamma}^{-4}+\ell_{\sigma}^{-2} q^{2}+q^{4}\left(1+b_{6}^{2} q^{2}+b_{8}^{4} q^{4}\right)\right]|h(\mathbf{q})|^{2} .
$$

Here $L$ is the lateral extension of the membrane; $b_{6}=\left(\kappa_{6} / \kappa\right)^{1 / 2}, b_{8}=\left(\kappa_{8} / \kappa\right)^{1 / 4}$ are nanometric lengths of the order of the thickness of the membrane, and $\ell_{\gamma}=(\kappa / \gamma)^{1 / 4}, \ell_{\sigma}=(\kappa / \sigma)^{1 / 2}$ are very large lengths in the case of free membranes with a vanishing tension.

To describe the coupling of the membrane with a pointwise isotropic inclusion labeled $i$, situated in $\mathbf{r}=\mathbf{r}_{i}$, we generalize the expression used in Ref. [1] (in which the term proportional to $\bar{D}$ below was omitted):

$$
U_{i}=\int d x d y \delta\left(\mathbf{r}-\mathbf{r}_{i}\right)\left[A \nabla^{2} h(\mathbf{r})-\frac{D}{2} \partial_{\alpha} \partial_{\beta} h(\mathbf{r}) \partial_{\alpha} \partial_{\beta} h(\mathbf{r})-\frac{\bar{D}}{2} \partial_{\alpha} \partial_{\alpha} h(\mathbf{r}) \partial_{\beta} \partial_{\beta} h(\mathbf{r})\right],
$$

where $\alpha, \beta \in\{1,2\}$ stand for the $x$ and $y$ coordinates and $\delta(\mathbf{r})$ is the Dirac distribution. Summation over repeated indices will be implied throughout. For a membrane containing two isotropic inclusions, the total energy functional is $F_{\mathrm{m}}+U_{1}+U_{2}$. To proceed, we rewrite the interaction terms $U_{i}$, up to a constant, as

$$
U_{i}=\frac{\Gamma}{2}\left(H_{\alpha \beta}^{(i)}-C_{\alpha \beta}^{(i)}\right)^{2}+\frac{\Gamma \epsilon}{2} H_{\alpha \alpha}^{(i)} H_{\beta \beta}^{(i)},
$$

where $H_{\alpha \beta}^{(i)} \equiv \partial_{\alpha} \partial_{\beta} h\left(\mathbf{r}_{i}\right)$, and $C_{\alpha \beta}^{(i)}=c_{0} \delta_{\alpha \beta}$ for an isotropic inclusion. The constants $\Gamma, \epsilon$ and $c_{0}$ are given by $\Gamma=-D$, $\epsilon=\bar{D} / D$ and $c_{0}=A / D$. Note that the condition $\epsilon>-\frac{1}{2}$ has to be satisfied, overwise the membrane would be locally unstable. In order to give a simple interpretation of the interaction, we can also write Eq. (4) in term of the principal values $H_{1}^{(i)}$ and $H_{2}^{(i)}$ of the local curvature tensor $H_{\alpha \beta}^{(i)}$, i.e.,

$$
U_{i}=\frac{1}{2}(1+2 \epsilon) \Gamma\left[\left(H_{1}^{(i)}-c\right)^{2}+\left(H_{2}^{(i)}-c\right)^{2}\right]-\frac{1}{2} \Gamma \epsilon\left(H_{1}^{(i)}-H_{2}^{(i)}\right)^{2} .
$$

The first two terms show that the inclusion locally promote an isotropic curvature $c$, and the third term distinguishes conformations for which the deviation of $H_{1}^{(i)}$ and $H_{2}^{(i)}$ from $c$ have identical or opposite signs. Note that it has been implicitely assumed that $\Gamma>0$.

In order to calculate the interaction energy between the two inclusions, we must minimize $F_{\mathrm{m}}+U_{1}+U_{2}$ with respect to the shape of the membrane. We do this in two steps: (i) first, we minimize $F_{\mathrm{m}}$ with respect to the membrane shape while setting prescribed values of $H_{\alpha \beta}^{(i)}$, which yields $F_{\min }\left(H_{\alpha \beta}^{(1)}, H_{\alpha \beta}^{(2)}\right)$, (ii) next, we minimize $F_{\text {tot }}=$ $F_{\min }\left(H_{\alpha \beta}^{(1)}, H_{\alpha \beta}^{(2)}\right)+U_{1}+U_{2}$ with respect to $H_{\alpha \beta}^{(1)}$ and $H_{\alpha \beta}^{(2)}$.

The first step was performed in Ref. [6]. The result is

$$
F_{\min }\left(H_{\alpha \beta}^{(1)}, H_{\alpha \beta}^{(2)}\right)=\frac{1}{2} \kappa \mathrm{K}^{\mathrm{t}} \mathrm{M}^{-1} \mathrm{~K} .
$$

In this expression, the subscript $t$ means transpose,

$$
\mathrm{K}^{\mathrm{t}}=\left(H_{11}^{(1)}, H_{12}^{(1)}, H_{22}^{(1)}, H_{11}^{(2)}, H_{12}^{(2)}, H_{22}^{(2)}\right),
$$

$\mathrm{M}$ is the $6 \times 6$ matrix:

$$
\mathbf{M}=\left(\begin{array}{cccccc}
G_{1111}(0) & G_{1112}(0) & G_{1122}(0) & G_{1111}(\mathbf{r}) & G_{1112}(\mathbf{r}) & G_{1122}(\mathbf{r}) \\
G_{1112}(0) & G_{1122}(0) & G_{1222}(0) & G_{1112}(\mathbf{r}) & G_{1122}(\mathbf{r}) & G_{1222}(\mathbf{r}) \\
G_{1122}(0) & G_{1222}(0) & G_{2222}(0) & G_{1122}(\mathbf{r}) & G_{1222}(\mathbf{r}) & G_{2222}(\mathbf{r}) \\
G_{1111}(-\mathbf{r}) & G_{1112}(-\mathbf{r}) & G_{1122}(-\mathbf{r}) & G_{1111}(0) & G_{1112}(0) & G_{1122}(0) \\
G_{1112}(-\mathbf{r}) & G_{1122}(-\mathbf{r}) & G_{1222}(-\mathbf{r}) & G_{1112}(0) & G_{1122}(0) & G_{1222}(0) \\
G_{1122}(-\mathbf{r}) & G_{1222}(-\mathbf{r}) & G_{2222}(-\mathbf{r}) & G_{1122}(0) & G_{1222}(0) & G_{2222}(0)
\end{array}\right)
$$


in which $\mathbf{r}=\mathbf{r}_{1}-\mathbf{r}_{2}$, and $G_{\alpha \beta \gamma \delta}(\mathbf{r})=\partial_{\alpha} \partial_{\beta} \partial_{\gamma} \partial_{\delta} G(\mathbf{r})$, where

$$
G(\mathbf{r})=\int \frac{d^{2} q}{(2 \pi)^{2}} \frac{e^{i \mathbf{q} \cdot \mathbf{r}}}{\ell_{\gamma}^{-4}+\ell_{\sigma}^{-2} q^{2}+q^{4}\left(1+b_{6}^{2} q^{2}+b_{8}^{4} q^{4}\right)}
$$

is the Green function associated with the elastic Hamiltonian. Note that no cutoff is required for the convergence of $G_{\alpha \beta \gamma \delta}(0)$ thanks to terms of order $q^{6}$ and $q^{8}$. The question of the high-wavevector cutoff will be raised later.

To perform the second step of the minimization, we rewrite the interaction term as

$$
U_{1}+U_{2}=\frac{\Gamma}{2}(\mathrm{~K}-\mathrm{C})^{\mathrm{t}} \mathrm{N}(\mathrm{K}-\mathrm{C})
$$

where

$$
\mathrm{C}^{\mathrm{t}}=\left(C_{11}^{(1)}, C_{12}^{(1)}, C_{22}^{(1)}, C_{11}^{(2)}, C_{12}^{(2)}, C_{22}^{(2)}\right)
$$

contains the curvatures attempted to be set by the inclusions, and $\mathrm{N}$ is the $6 \times 6$ block-diagonal matrix:

$$
\mathrm{N}=\left(\begin{array}{ccc}
1+\epsilon & 0 & \epsilon \\
0 & 2 \epsilon & 0 \\
\epsilon & 0 & 1+\epsilon
\end{array}\right) \otimes\left(\begin{array}{ll}
1 & 0 \\
0 & 1
\end{array}\right)
$$

where $\otimes$ stands for the matrix direct product.

The resulting total energy $F_{\text {tot }}=F_{\min }+U_{1}+U_{2}$ is quadratic in $\mathrm{K}$, and its minimization yields

$$
F_{\text {tot, } \min }=-\frac{\Gamma^{2}}{2} \mathrm{C}^{\mathrm{t}} \mathrm{N}\left[\kappa \mathrm{M}^{-1}+\Gamma \mathrm{N}\right]^{-1} \mathrm{NC}+\frac{\Gamma}{2} \mathrm{C}^{\mathrm{t}} \mathrm{NC}
$$

After some algebra, this expression can be recast in the simpler form, similar to that of Eq. (6) :

$$
F_{\text {tot, } \min }=\frac{1}{2} \kappa \mathrm{C}^{\mathrm{t}}\left(\mathrm{M}+\frac{\kappa}{\Gamma} \mathrm{N}^{-1}\right)^{-1} \mathrm{C} \text {. }
$$

Equation (14) is very general and corresponds to our central result: it describes the interaction between two pointwise inclusions to all orders in their inverse separation $1 / r$ (as long as the Hamiltonian $F_{m}$ is fit to describe the membrane distortion energy at the scale $r$ ), for any value of the rigidities $\Gamma$ and $\Gamma \epsilon$, and actually for isotropic or anisotropic inclusions, since the above derivation does not assume any form for the components of C. For anisotropic inclusions, C should contain the components of the curvature tensor favored by the inclusions [6].

To proceed further, we must first determine the components of the matrix M. In the large $r$ limit, i.e., $r \gg b_{6}, b_{8}$ (recall that $b_{6}$ and $b_{8}$ are nanoscopic lengths), and assuming also $r \ll \ell_{\gamma}, \ell_{\sigma}$ (i.e., taking $\ell_{\gamma} \rightarrow \infty$ and setting strictly $\ell_{\sigma}^{-1}=0$ for the sake of simplicity), Eq. (9) yields

$$
G(\mathbf{r}) \simeq C_{1}+\frac{1}{16 \pi} r^{2} \ln \left(\frac{r^{2}}{C_{2}}\right)
$$

The constants $C_{1}$ and $C_{2}$ depend on $\ell_{\gamma}$, but they actually disappear in the calculation of $G_{\alpha \beta \gamma \delta}(\mathbf{r})$, leaving $1 /(16 \pi)\left(x^{2}+\right.$ $\left.y^{2}\right) \ln \left(x^{2}+y^{2}\right)$ as the only relevant contribution in $G(\mathbf{r})$. To calculate the components of the matrix $\mathrm{M}$ in the large $r$ limit defined above, we set $x=r$ and $y=0$, yielding, $G_{1111}=G_{1122} \simeq-1 /\left(4 \pi r^{2}\right), G_{2222} \simeq 3 /\left(4 \pi r^{2}\right)$, and $G_{1112}=G_{1222}=0$. Next, we have

$$
G_{\alpha \beta \gamma \delta}(0)=n_{\alpha \beta \gamma \delta} \times \int \frac{d q}{16 \pi} \frac{q^{5}}{\ell_{\gamma}^{-4}+\ell_{\sigma}^{-2} q^{2}+q^{4}\left(1+b_{6}^{2} q^{2}+b_{8}^{4} q^{4}\right)} \equiv b^{-2} n_{\alpha \beta \gamma \delta},
$$

where $n_{\alpha \beta \gamma \delta}$ contains the angular part of the integration on $\mathbf{q}$. Simple calculations yield $n_{1111}=n_{2222}=3, n_{1122}=1$, and $n_{1112}=n_{1222}=0$. For $b_{6} \approx b_{8} \ll \ell_{\gamma}, \ell_{\sigma}$, as previously specified, the constant $b$ is a nanometric length comparable to $b_{6}$ or $b_{8}$. Therefore, without loss of generality, we have (still in the large $r$ limit):

$$
\mathrm{M} \simeq \frac{1}{b^{2}}\left(\begin{array}{lll}
3 & 0 & 1 \\
0 & 1 & 0 \\
1 & 0 & 3
\end{array}\right) \otimes\left(\begin{array}{ll}
1 & 0 \\
0 & 1
\end{array}\right)+\frac{1}{4 \pi r^{2}}\left(\begin{array}{ccc}
-1 & 0 & 1 \\
0 & -1 & 0 \\
-1 & 0 & 3
\end{array}\right) \otimes\left(\begin{array}{ll}
0 & 1 \\
1 & 0
\end{array}\right)
$$


To calculate the interaction $F_{\text {int }}(r)$ between two identical isotropic inclusions, we set

$$
\mathrm{C}^{\mathrm{t}}=(c, 0, c, c, 0, c),
$$

and, using Eq. (14), we obtain:

$$
F_{\text {int }}(r)=\frac{\kappa \Gamma^{3} c^{2}(1+2 \epsilon)^{2}}{2 \pi^{2}\left(\kappa+2 \Gamma b^{-2}\right)\left[\kappa+4 \Gamma b^{-2}(1+2 \epsilon)\right]^{2}} \frac{1}{r^{4}}+\mathcal{O}\left(\frac{1}{r^{6}}\right) .
$$

We now consider two limits: hard inclusions, corresponding to $\Gamma / \kappa \gg b^{2}$, and soft inclusions, corresponding to $\Gamma / \kappa \ll b^{2}$ (note that we keep $\epsilon$ of order unity for the sake of simplicity). In the former case, the inclusions strongly set their preferred curvature; in the latter case, they only weakly tend to establish it.

For hard inclusions, we let $\Gamma$ go to infinity, and to leading order in $1 / r$, we obtain

$$
F_{\mathrm{int}, \text { hard }}(r) \simeq \frac{\kappa b^{2} c^{2}}{64 \pi^{2}}\left(\frac{b}{r}\right)^{4} .
$$

We thus recover the form obtained in Refs. [2, 3, 4, 5, 6], with $\kappa$ appearing in the numerator. Here the microscopic length $b$ arising from the membrane Hamiltonian plays the role of the cutoff introduced in Refs. [3, 4, [5, [6], or the role of the radius of the disk modeling the inclusion in the case of Ref. 2].

For soft inclusions, we obtain to leading order in $1 / r$ and at lowest-order in $\Gamma$ (like in Ref. 1]):

$$
F_{\text {int }, \text { soft }}(r) \simeq \frac{\Gamma^{3} c^{2}}{2 \pi^{2} \kappa^{2} r^{4}}(1+2 \epsilon) \equiv-\frac{2 A^{2} D}{4 \pi^{2} \kappa^{2} r^{4}}(1+2 \epsilon),
$$

where $\kappa$ appears now to the second power in the denominator. This expression coincides exactly with the Eq. (37) of Ref. [1], in which $\epsilon=0$ was implicitely assumed. Contrary to the case of hard inclusions, the microscopic length $b$ does not appear in the interaction, it would however reappear in the next order term $\mathcal{O}\left(\Gamma^{4}\right)$. We recall that $1+2 \epsilon$ is positive, hence the interaction is always repulsive in the present case of isotropic inclusions.

We have therefore shown that there is no fundamental discrepancy between the recent result of Marchenko and Misbah [1] and the earlier results published in Refs. 2, 3, 4, 5, 6]. The result of Ref. 1] applies to soft inclusions, i.e., to inclusions that weakly tend to curve the membrane, while the result of Refs. 2, 3, 4, 5, 6] is valid for hard inclusions that strongly enforce their preferred membrane curvature.

\section{THE ROLE OF THE CUT-OFF}

Let us now comment on the role of the high-wavevector cutoff. Of course, any well-defined elastic theory does involve a coarse-graining cutoff $a^{-1}$, and the elastic constants $\kappa(a), \kappa_{6}(a)$, and $\kappa_{8}(a)$ depend on it. Thanks to the high-order bending elastic constants $\kappa_{6}$ and $\kappa_{8}$, there was no need to introduce a cutoff to calculate the interaction between the quadratic pointwise inclusions considered here. This is however somewhat arbitrary, since the microscopic length $b$ arising from this procedure is expected to compare with the natural cutoff $a$. This simply means that reintrocuding the cutoff would merely redefine $b$, leaving its order of magnitude unchanged. It is therefore unimportant to specify whether the cutoff was introduced or not in the procedure defining the microscopic length $b$ that appears in the interaction.

\section{COARSE-GRAINING OF THE MEMBRANE-INCLUSION SYSTEM}

Describing an inclusion by a pointwise potential is of course a mathematical approximation hiding some physical microscopic length-scale. One may actually choose the "resolution" of the pointwise description, i.e., the high-wavevector cutoff, by using a renormalization procedure. For instance, one may choose a "microscopic" cutoff comparable with the inverse inclusion size, or a much smaller cutoff corresponding to an elementary point size much larger than the inclusion. The question then naturally arises of how the constants of the pointwise quadratic potential will depend on the coarse-graining scale.

Let us therefore consider a membrane described with an upper wavevector cutoff $\Lambda=a^{-1}$. The membrane hosts a single pointwise inclusion located at $\mathbf{r}=\mathbf{r}_{1}$. We shall perform a coarse-graining of this system in order that the new wavevector cutoff becomes $\Lambda^{\prime}=(\mu a)^{-1}<\Lambda$. We assume the whole system to be in thermal equilibrium at the 
temperature $T$, however, since all energies are quadratic, the renormalization will be athermal and we can set $k_{\mathrm{B}} T=1$ without loss of generality. The partition function can then be written as

$$
\mathcal{Z}=\int \mathcal{D} h \exp \left(-F_{\mathrm{m}}[h]-U_{1}[h]\right)
$$

where the inclusion's pointwise potential can be written as $U_{1}=\frac{1}{2} \Gamma\left(\mathrm{K}_{1}-\mathrm{C}_{1}\right)^{\mathrm{t}} \mathrm{N}_{1}\left(\mathrm{~K}_{1}-\mathrm{C}_{1}\right)$, as in Eq. (10). Here, $\mathrm{N}_{1}$ is the $3 \times 3$ uper-left block of the matrix $\mathrm{N}$ of Eq. (12), and $\mathrm{K}_{1}^{\mathrm{t}}=\left(H_{11}^{(1)}, H_{12}^{(1)}, H_{22}^{(1)}\right), \mathrm{C}_{1}^{\mathrm{t}}=(c, 0, c)$. It is convenient to write $\exp \left(-U_{1}\right)$ as the result of a Gaussian integral (Hubbard-Stratonovich transformation [9]):

$$
\exp \left(-U_{1}\right) \propto \int d^{3} \Phi \exp \left[-\frac{1}{2} \Phi^{\mathrm{t}} \mathrm{N}_{1}^{-1} \Phi+i \Phi^{\mathrm{t}}\left(\mathrm{C}_{1}-\int \frac{d^{2} q}{(2 \pi)^{2}} \mathrm{~K}_{1}(\mathbf{q}) e^{i \mathbf{q} \cdot \mathbf{r}_{1}}\right)\right]
$$

where $\mathrm{K}_{1}{ }^{\mathrm{t}}(\mathbf{q})=\left(-q_{1}^{2} h(\mathbf{q}),-q_{1} q_{2} h(\mathbf{q}),-q_{2}^{2} h(\mathbf{q})\right)$. Then, going to the variables $h(\mathbf{q})$, Eq. (22) becomes (up to a multiplicative constant):

$$
\mathcal{Z}=\int d^{3} \Phi \exp \left(-\frac{1}{2} \Phi^{\mathrm{t}} \mathrm{N}_{1}^{-1} \Phi+i \Phi^{\mathrm{t}} \mathbf{C}_{1}\right) \int \prod_{q<1 / a} d h(\mathbf{q}) \exp \left[-\int \frac{d^{2} q}{(2 \pi)^{2}} \frac{\kappa}{2} q^{4}|h(\mathbf{q})|^{2}+i \Phi^{\mathrm{t}} \mathrm{K}_{1}(\mathbf{q}) e^{i \mathbf{q} \cdot \mathbf{r}_{1}}\right]
$$

Since the argument of the second exponential in the above equation is a quadratic function of $h(\mathbf{q})$, it is straightforward to integrate over the $h(\mathbf{q})$ with wavevectors in the range $(\mu a)^{-1}<|\mathbf{q}|<a^{-1}$. Once this integration is performed, the resulting integral over $\Phi$ is Gaussian, and yields, after some elementary algebra,

$$
\mathcal{Z}=\int \mathcal{D} h^{<} \exp \left(-F_{m}\left[h^{<}\right]-U_{1}^{\mathrm{eff}}\left[h^{<}\right]\right)
$$

where $h^{<}(\mathbf{r})=(2 \pi)^{-2} \int_{q<1 /(\mu a)} d^{2} q h(\mathbf{q}) e^{i \mathbf{q} \cdot \mathbf{r}}$, and

$$
\begin{aligned}
U_{1}^{\mathrm{eff}} & =\frac{\Gamma^{\mathrm{eff}}}{2}\left(\partial_{\alpha} \partial_{\beta} h^{<}\left(\mathbf{r}_{1}\right)-C_{\alpha \beta}^{(1)}\right)^{2}+\frac{\Gamma^{\mathrm{eff}} \epsilon^{\mathrm{eff}}}{2} \partial_{\alpha} \partial_{\alpha} h^{<}\left(\mathbf{r}_{1}\right) \partial_{\beta} \partial_{\beta} h^{<}\left(\mathbf{r}_{1}\right) \\
\Gamma^{\mathrm{eff}} & =\Gamma \frac{\kappa b_{0}^{2}}{\kappa b_{0}^{2}+2 \Gamma\left(1-\mu^{-2}\right)} \\
\epsilon^{\mathrm{eff}} & =\frac{\epsilon \kappa b_{0}^{2}+\Gamma(1+2 \epsilon)\left(\mu^{2}-1\right)}{\kappa b_{0}^{2} \mu^{2}-4 \Gamma(1+2 \epsilon)\left(\mu^{2}-1\right)}
\end{aligned}
$$

where we have defined $b_{0}=4 \sqrt{2 \pi} a$. This set of exact equations is our second main result. It describes the effective quadratic coupling between the inclusion and the membrane in the coarse-grained pointwise description corresponding to the new cutoff $(\mu a)^{-1}$. Note that the inclusion's preferred curvature $C_{1}$ is unaffected by the coarse-graining procedure. This result may seem surprising, however one should realize that the actual curvature set by the inclusion will naturally be smaller in the coarse-grained description due to the fact that $\Gamma^{\mathrm{eff}}<\Gamma$.

In principle, the interaction between two inclusions should be independent of the level of coarse-graining chosen to describe the inclusions (as long as their separation is larger than the length-scale of the cutoff). One can indeed check that the interaction Eq. (19) is unchanged under the substitutions $b \equiv b_{0} \rightarrow \mu b_{0}, \Gamma \rightarrow \Gamma^{\mathrm{eff}}$ and $\epsilon \rightarrow \epsilon^{\mathrm{eff}}$.

Now, since the rigidities $\Gamma$ and $\epsilon$ are scale-dependent, an inclusion may be hard or soft depending on the coarsegraining level. Indeed, according to the invariance of the interaction mentioned above, the criterion for the hardness or softness of the inclusion is to compare $\Gamma^{\mathrm{eff}} / \kappa$ to $\left(\mu b_{0}\right)^{2}$. For $\mu \gg 1$, the "rigidity" $\Gamma /\left(\kappa b_{0}^{2}\right)$, which becomes $\Gamma^{\mathrm{eff}} /\left(\kappa \mu^{2} b_{0}^{2}\right)$ after coarse-graining, is reduced by a factor $\simeq \mu^{2}\left[1+2 \Gamma /\left(\kappa b_{0}^{2}\right)\right] \gg 1$. Hence all inclusions effectively become soft at large coarse-graining scales.

Is this in contradiction with the fact that hard inclusions have an interaction proportional to $\kappa$ while soft inclusions have an interaction inversely proportional to $\kappa^{2}$ ? The answer is no, because the effective constants $D$ and $A$ introduced by Misbah et al. [1] actually depend on $\kappa$. (This is another way to see that there is no discrepancy between the results of Ref. 1] and those of the previous works.) Indeed, starting from a hard inclusion $\left(\Gamma \gg \kappa b_{0}^{2}\right)$, we obtain, in the limit $\mu \gg 1$ where all inclusions become soft, $\Gamma^{\mathrm{eff}} \simeq \frac{1}{2} \kappa b_{0}^{2}$ and $\epsilon^{\mathrm{eff}} \simeq-\frac{1}{4}$, while the preferred curvature $c_{0}$ is unchanged. With the correspondance $D^{\mathrm{eff}}=-\Gamma^{\mathrm{eff}}$ and $A^{\mathrm{eff}}=c_{0} \Gamma^{\mathrm{eff}}$, we obtain $\left(A^{\mathrm{eff}}\right)^{2} D^{\mathrm{eff}} \propto \kappa^{3}$, hence Eq. (21) indeed yields an interaction proportional to $\kappa$.

We therefore conclude that the correct procedure to determine whether a given inclusions is fundamentally hard or soft is to estimate $\Gamma$ at the coarse-graining level where the cutoff $a$ compares with the inclusion size. The corresponding "microscopic" value $\Gamma_{m}$ must then, according to the discussion at the end of Sec. II be compared with $\kappa a^{2}$ (since $b$ and $a$ are expected to be of the same order of magnitude). An inclusion will be fundamentally hard if $\Gamma_{m} \gg \kappa a^{2}$ or fundamentally soft if $\Gamma_{m} \ll \kappa a^{2}$. 


\section{PROTEINS AS "HARD" INCLUSION}

We now argue that integral proteins of conical shape should be fundamentally considered as hard inclusions. First, it is very likely that the membrane elasticity is too weak to alter the conical shape of the protein, because of the strong dipolar interactions within the bundles of $\alpha$-helices in the transmembrane domain. Beside, a significant change in the protein shape would most probably alter its function. We can therefore assume that the protein behaves as a rigid conical body and that the weakness of the curvature potential embodied in the parameter $\Gamma_{m}$ arises from the tilt degree of freedom of the lipids. Let us consider a conical protein having a circular section of radius $R$ in the membrane plane and an aperture angle $2 \theta$. An estimate of $\Gamma_{m}$ can be obtained by calculating the energy stored in the lipid tilt when one assumes that the membrane midplane remains perfectly flat. The latter is $W \approx B \theta^{2} \times 2 \pi R \times \xi$, where $B$ is the elastic constant associated with the tilt and $\xi \approx(\kappa / B)^{1 / 2}$ the tilt relaxation length. With $c_{0}=\theta / R$, which corresponds to the membrane curvature that the protein attempts to set, this energy can be rewritten as $W \approx \frac{1}{2} \Gamma_{m} c_{0}^{2}$ with $\Gamma_{m} \approx 4 \pi \kappa R^{3} / \xi$. Hence we obtain

$$
\frac{\Gamma_{m}}{\kappa a^{2}} \approx 4 \pi \frac{R^{3}}{a^{2} \xi} .
$$

Interestingly, this quantity is independent of $\kappa$ and it grows as $R^{3}$. With typically $R \simeq 6 \mathrm{~nm}, a \simeq \xi \simeq 3 \mathrm{~nm}$, we obtain $\Gamma_{m} /\left(\kappa a^{2}\right) \approx 100$, which justifies treating conical proteins as a hard inclusion.

\section{CONCLUSION}

We have calculated the induced interaction acting between pointwise membrane inclusions that locally interact up to quadratic order with the membrane curvature tensor. According to the rigidity of the potential constraining the local membrane curvature, inclusions can be viewed as hard or soft. The interaction between hard inclusions is proportional to $\kappa$ while the interaction between soft inclusions is inversely proportional to $\kappa^{2}$ (both are also inversely proportional to the fourth power of the separation in the case of isotropic inclusions). This reconciliates the recent view of Marchenko and Misbah [1] with those of earlier works.

To discriminate whether a given inclusion is fundamentally hard or soft, one should however consider the coupling constants defined at the microscopic level, i.e., defined in a pointwise description in which the elementary point-size (the cutoff of the model) compares with the inclusion size. Indeed, we have shown that coarse-graining the pointwise description to length-scales much larger that the inclusion's size transforms any hard inclusion into a soft one, however with coupling constants depending on $\kappa$ in such a way that the global interaction remains proportional to $\kappa$ if the inclusions was fundamentally hard. In the light of this analysis we have shown that transmembrane proteins should be considered as fundamentally hard inclusions.

\section{Acknowledgments}

Helpful interactions with Paolo Galatola are gratefully acknowledged.

[1] V. I. Marchenko, C. Misbah, Eur. Phys. J. E 8, 477 (2002).

[2] M. Goulian, R. Bruinsma, P. Pincus, Europhys. Lett. 22, 145 (1993).

[3] J. M. Park, T. C. Lubensky, J. Phys. I 6, 1217 (1996).

[4] K. S. Kim, J. Neu, G. Oster, Biophys. J. 75, 2274 (1998).

[5] P. G. Dommersnes, J.-B. Fournier, Eur. Phys. J. B 12, 9 (1999).

[6] P. G. Dommersnes, J.-B. Fournier, to be published in Biophys. J. (2002).

[7] W. Helfrich, Z. Naturforsch. 28c, 693 (1973).

[8] R. Goetz, W. Helfrich, J. Phys. II France 6, 215 (1996).

[9] P. M. Chaikin and T. C. Lubensky, Principles of Condensed Matter Physics, Cambridge University Press. 